\title{
Torsional Vibration of Multi-Plate Clutches Analaysed WiTh Hybrid MeTHOD
}

\author{
BELSAK, A. \& HIRZ, M.
}

Abstract: Multi-plate clutches for automotive drivetrains have the following advantages if compared to conventional single plate clutches. On the other hand, they require more space in axial direction for installation, whereby the number of frictionand steel-plates influences the transmittable torque behaviour. As it comes to friction in guide-ways, axial forces are reduced from one plate to another, which lowers the total torque transmission potential and influences the dynamic behaviour. This leads to different torsional vibration signatures under typical operating conditions and to special requirements on the applied analysis methods. In this context, the paper introduces a new approach of a hybrid adaptive analysis method of vibrations produced in non-stationary processes with the target to support evaluation of the operation and to improve control quality in different applications.

Key words: Multi-plate clutch, friction, vibration, hybrid analysis
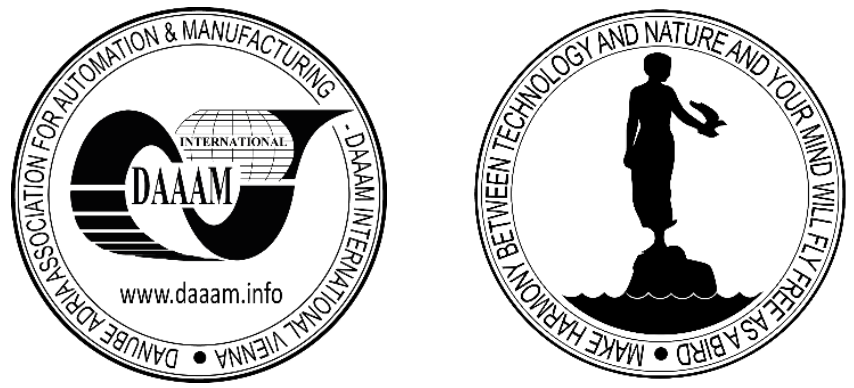

Authors' data: Assist. Prof. Belsak, A[les]*; Assoc. Prof. Hirz, M[ario]**, * University of Maribor, Faculty of mechanical Engineering, Smetanova 17, SI-2000, Maribor, Slovenia, ales.belsak@um.si ** Graz University of Technology, Institute of Automotive Engineering, Inffeldgasse 11/II, A-8010, Graz, Austria, mario.hirz@tugraz.at

This Publication has to be referred as: Belsak, A[les] \& Hirz, M[ario] (2019). Torsional Vibration of Multi-Plate Clutches Analaysed with Hybrid Method, Chapter 13 in DAAAM International Scientific Book 2019, pp.175-190, B. Katalinic (Ed.), Published by DAAAM International, ISBN 978-3-902734-24-2, ISSN 1726-9687, Vienna, Austria

DOI: $10.2507 /$ daaam.scibook.2019.13 
Belsak, A. \& Hirz, M.: Torsional Vibration of Multi-Plate Clutches Analaysed with...

\section{Introduction}

Types and purposes of friction clutches in different kinds of vehicles vary in a broad range. In general, they are used to transmit torque from an input to an output shaft in a controlled way by friction between compressed plates, which have specifically designed linings. Different friction linings and materials come to use with the target to achieve a suitable friction combination and the desired behaviour; this applies primarily to the coefficient of friction, noise vibration and harshness (NVH), and wear. The application of multi-plate clutches often improves the transmittable torque behaviour, whereas the installation space is just slightly larger in comparison to single-plate clutch systems.

Fig. 1 shows an exemplary design of conventional multi-plate clutches. While the clutch is compressed, torque is transmitted by friction occurring between the individual external (steel) and internal (friction) plates. The external plates, which are guided in the outer plate carrier, slide axially but they cannot rotate to the outer plate carrier. The internal plates are guided in the inner plate carrier and can also slide axially; however, they cannot rotate in relation to the inner plate carrier. When the pressure plate (operating piston) is closed, the clutch pack is pressed together. In this example, load is placed on the piston by means of an electrohydraulic actuation, however, also mechanical, pneumatic, and electromagnetic actuations are present. When there is no load acting anymore, a return spring pushes the piston back with a mechanism and the clutch opens. (Gradwohl, 2017) (Belsak\& Hirz,2018)

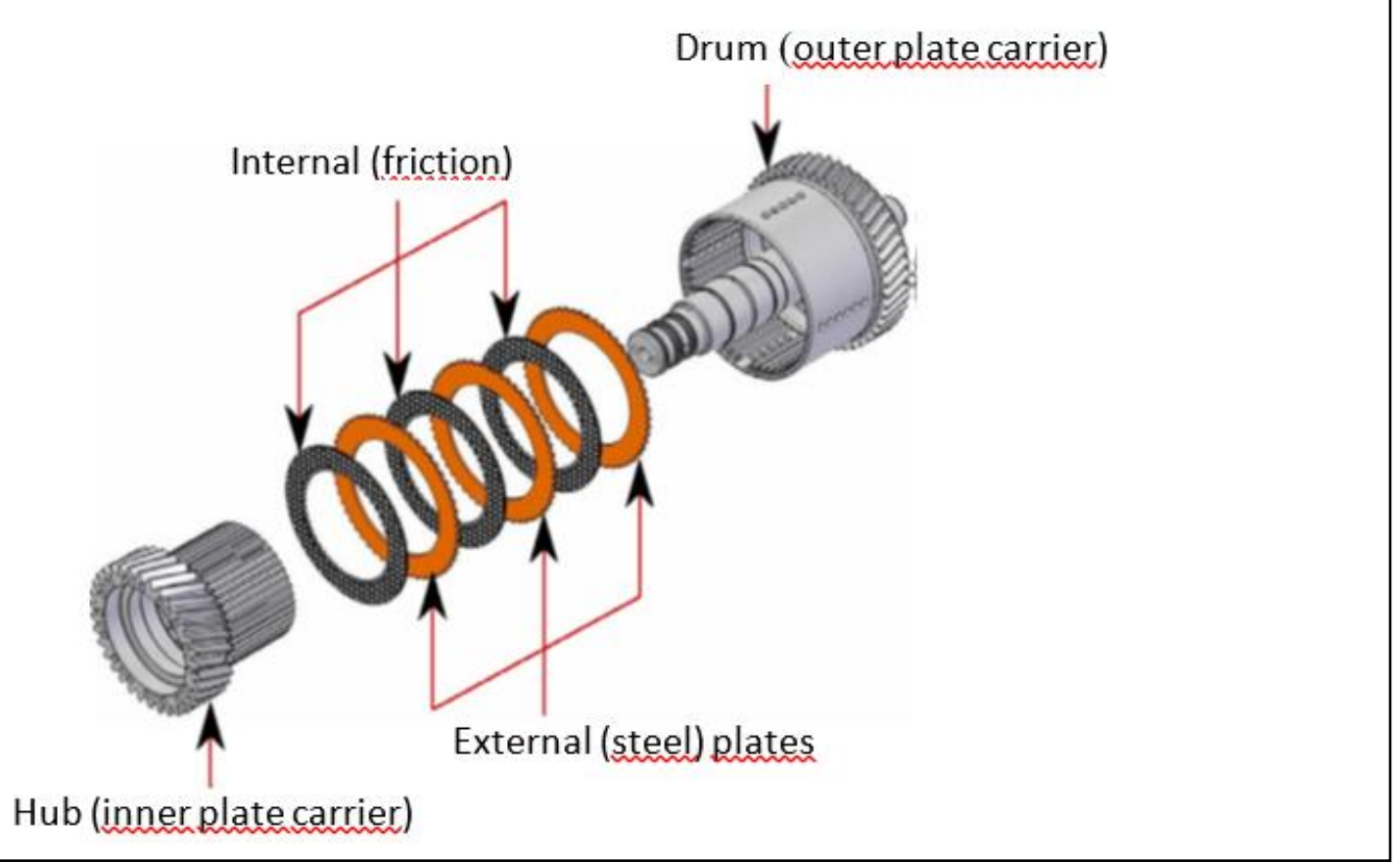

Fig. 1. Conventional multi-plate clutch [2]

The main parameter for calculation of the transmittable torque include the coefficient of friction, the normal forces in each friction pairing, the number of friction surfaces, and the mean friction radius. 
Considering a simplification of equal pressure and a constant coefficient of friction distribution, the inner and outer radius of the friction linings only have an impact upon the mean friction radius. The axial normal force in each lining can be represented as a function of the acting force under consideration of the friction behaviour in the guides of the external and internal plates. (Gradwohl, 2017)

Multi-plate clutches are designed as wet or dry clutches. In case of wet clutches, oil is used for cooling and for enhancing $\mathrm{NVH}$ and wear behaviour of the linings. (Dolcini et al., 2010) Relatively high drag losses are caused by oil, particularly when the clutch is open. Dry clutches that work without oil can be used to avoid this effect. (Orthwein, 2004) (Butlin \& Woodhause, 2009) The coefficients of friction of dry clutches are usually higher; consequently, their torque transmission potential is higher. As a result, it is possible to decrease the axial normal force, the number of friction surfaces and/or the radial expansion of dry clutches to achieve the same torque transmission potential. However, when developing dry clutches, different challenges regarding thermal behaviour, $\mathrm{NVH}$ and wear have to be dealt with. (Popov, 2010) (Gang, 2014) As it is of special concern, the behaviour of interaction of friction and vibration of the clutch system were dealt by several researchers, e.g. (Sergienko \& Bukharov, 2015) (Ouyang \& Motterhead, 2001) (Qiao \& Ibrahim, 1999). In this context, the analysis of torsional vibration is of great interest for investigation of the NHV signature. (Centea et al., 2001) (Chengwu \& Rajendra, 2009) The introduced various methods for determining operating parameters are very challenging in terms of effort and complexity (He et al., 2011) (Lingestena et al., 2012). In addition, the optimisation of actuators design and clutch control represent another mechatronic challenge, which requires high effort (Pinte et al., 2010) (Meng et al., 2015). In this way, the development of new approaches of modelling dynamic behaviour of multiplate clutches is required to support effective clutch design under consideration of the interaction between friction and vibrations behaviour (Butlin \& Woodhause, 2009) (Ibrahim et al., 2000).

\section{Time dependent signal analysis}

Factors that have a negative impact upon the reliability and adequate quality of operation are as follows (in the specified order): the presence of mechanical defects, effects caused by wear, tear and thermal conditions of plates, eccentricity caused by backlash in housing and errors when assembling and manufacturing the clutch. A suitable method for monitoring the conditions of a clutch - specifically the vibrationand torque behaviour - is presented in the following sections. Based on reference values, measured deviations of the frequency spectrum deliver information about potential functional errors. A clutch is a complex mechanical system and as such, it has changeable dynamic reactions. Consequently, variations of frequency-relevant components cannot be defined in time, and the approach based on time-frequency methods is more appropriate. A clutch represents a typical nonlinear and nonstationary dynamical system and, consequently, an analysis with time changing sensitivity must be used. 
Frequency analysis is often used in diagnostics applications, but it yields good results only for periodical processes without local changes. When it comes to classical frequency analysis, time description of vibration is transformed into frequency description, and changes within a signal are averaged within the entire time period observed. This means that local changes are actually lost in the average of the entire function of vibration and thus they can be identified only with great difficulty or even not at all. With the time-frequency analysis, these disadvantages can be avoided. Local changes that differ from the global periodical oscillation are indicated by the appearance or disappearance of individual frequency components in a spectrogram. Thus, a signal is presented simultaneously in time and frequency.

In technical diagnostics, individual frequency components in signals are often present from time to time only. With time-frequency analysis it can be described in which way frequency components of non-stationary signals change with time and also it is possible to define their intensity levels. Typical representatives of time-frequency algorithms are Fourier, wavelet transforms and Gabor expansion. All linear transformations basically perform comparison of an elementary function that is defined in advance. Elementary functions of windowed Fourier and wavelet transforms are based on a time interval, and the time interval is restricted concerning the time frame. In this way, they are different from Fourier transform, where the elementary functions are not limited in relation to time. The resolution of frequency axis, used for window Fourier and wavelet transforms, includes time data in addition to frequency data.

Adaptive time-frequency transformation with spectral entropy is used for signal analysis. The unknown probability density function is estimated as the power density spectrum of a dynamics process. Applications of spectral estimation methods for probability density function estimation are used in relation to nonlinear signal processing, exemplary shown in (Pages-Zamora \& lagunas, 1995) (Pages-Zamora \& lagunas, 1999). Probability density function estimation is also discussed, e.g. in (Kay, 1998) (Bercher \& Vignat, 1999). The introduced model in the present publication makes it possible to compute the associated entropy of signals more effective, which represents an enhancement of state of the art methods.

\section{Adaptive method and spectral entropy analysis}

Although a clutch with all the elements enabling transmission of rotating movement represents a complex dynamic system, its movement is usually periodical. Faults and geometrical inaccuracies represent disturbing quantities and induce impulses, which can be indicated by local- and time-related changes in vibration signals. As a result, time-frequency changes can be observed, which are based on kinematics, dynamics and operating characteristics of the clutch system. Adaptive transform of signals was improved and concluded by Qian (Qian \& Chen, 1996) to a large extent, although different authors have developed algorithms without interference parts that reduce usability of individual transforms as opposed to Cohen's class. (Mertins, 1999) In this way, adaptive transform of a signal $x(t)$ can be expressed as follows: 


$$
x(t)=\sum_{p} B_{p} \cdot h_{p}(t)
$$

The following equations are used to determine analysis coefficients

$$
B_{p}=\left\langle x, h_{p}\right\rangle
$$

whereby similarity between the measured signal $x(t)$ and elementary functions $h_{p}(t)$ of transform is expressed.

A starting point with the parameter values $\mathrm{p}=0$ and $\mathrm{x}_{0}(\mathrm{t})=\mathrm{x}(\mathrm{t})$ is represented by the original signal. In the set of desired elementary functions, $h_{0}(t)$ has to be found, that is most similar to $\mathrm{x}_{0}(\mathrm{t})$ in the sense of

$$
\left|B_{p}\right|^{2}=\left.\max _{h_{p}}\left\langle x_{p}(t), h_{p}(t)\right\rangle\right|^{2}
$$

for $\mathrm{p}=0$. Afterwards, the remaining $\mathrm{x}_{1}(\mathrm{t})$ is calculated by

$$
x_{p+1}(t)=x_{p}(t)-B_{p} \cdot h_{p}(t)
$$

Although the generalisation idea is retained, $h_{p}(t)$ is to have a unit of energy representation of a signal. Consequently:

$$
\left\|h_{p}(t)\right\|^{2}=\frac{1}{2 \cdot \pi} \iint \sum_{p=0}^{n} h_{p}(t)=\lim _{h_{p}(t) \rightarrow f|\max |}=1
$$

The equation used to calculate the energy that is included in the remaining signal can be calculated as:

$$
\left\|\left.x_{p+1}(t)\right|^{2}=\right\| x_{p}(t) \|^{2}-\left|B_{p}\right|^{2}
$$

In the simulation process, equation (4) is repeated to find $\mathrm{h}_{1}(\mathrm{t})$ that would suit best $\mathrm{x}_{1}(\mathrm{t})$, etc. In each step one elementary function $h_{p}(t)$ that suits best $x_{p}(t)$ is found. Primarily, the objective of adaptive signal representation is to find a set of elementary functions $\left\{h_{p}(t)\right\}$ that are most similar to time-frequency structure of a signal and also are able to satisfy equations (1) and (2).

Expressing equation (5) as

$$
\left\|x_{p}(t)\right\|^{2}=\left\|x_{p+1}(t)\right\|^{2}+\left|B_{p}\right|^{2}
$$


Belsak, A. \& Hirz, M.: Torsional Vibration of Multi-Plate Clutches Analaysed with...

indicates that it is possible to determine the energy remaining in a signal at $\mathrm{p}$-th level on the basis of $\mathrm{p}+1$ level and a rest $\mathrm{B}_{\mathrm{p}}$. When continuing this process, the following result is acquired:

$$
\|x(t)\|^{2}=\sum_{p=0}^{\infty}\left|B_{p}\right|^{2}
$$

The equation is related to energy conservation. It is similar to Parseval's relation in the Fourier transform. When using the Wigner-Ville distribution for both sides of equation (1), the equations can be organised into two groups as follows:

$$
\begin{aligned}
P_{W V} x(t, \omega)= & \sum_{p} B_{p}^{2} \cdot P_{W V} h_{p}(t, \omega) \\
& +\sum_{p \neq q} B_{p} \cdot B_{q} * P_{W V}\left(h_{p}, h_{q}\right)(t, \omega)
\end{aligned}
$$

Elementary signal components represent the first group, whereas cross interference terms, which are ignored during the analysis, represent the second group

$$
\sum_{p \neq q} B_{p} \cdot B_{q}^{*} P_{W V}\left(h_{p}, h_{q}\right)(t, \omega) \Rightarrow 0
$$

As a result of the relation described in (8) and the given value of energy conservation, it is evident that

$$
\frac{1}{2 \cdot \pi} \cdot \iint \sum_{p \neq q} B_{p} \cdot B_{q}^{*} P_{W V}\left(h_{p}, h_{q}\right)(t, \omega)=0
$$

Because of this, it is possible to define a new time-dependent adaptive spectrum as:

$$
P_{A D T}(t, \omega)=\sum_{p}\left|B_{p}\right|^{2} \cdot P_{W V} h_{p}(t, \omega)
$$

In order to find a suitable elementary function $h_{p}(t)$ more effectively, the method of determining the signal entropy $\mathrm{S}(\mathrm{x})$ is used, after the selection of an appropriate elementary function $h_{p}(t)$ by determining analysis coefficients $B_{p}$. Thus, the suitable value of the entropy $S(x)$ of the signal $x(t)$ is found, which is calculated iteratively when searching for a suitable elementary function $h_{p}(t)$. If the value of the entropy does no longer change, the selected value of the elementary function is final.

Information entropy introduced by Shannon et al. is called Shannon entropy. Information entropy $S(x)$ of a random variable $X$ with the values $x_{1}, x_{2} \ldots x_{N}$ is defined as (Bercher \& Vignat, 2000): 


$$
S(x)=\sum_{i=1}^{n} p(x) \cdot \log _{a} \frac{1}{p\left(x_{i}\right)}=-\sum_{i=1}^{n} p(x) \cdot \log _{a} p\left(x_{i}\right), \quad a>1
$$

where $\mathrm{p}\left(\mathrm{x}_{\mathrm{i}}\right)$ are probability density functions or probabilities of acceptance by the random variable $X$ values $\mathrm{x}_{\mathrm{i}}$. A degree of uncertainty related with the occurrence of the result is a typical behaviour of Shannon entropy. The higher the value of the entropy, the more uncertain and more difficult to predict is the outcome. Generally speaking, the entropy of $\mathrm{X}$ is a measure of expected uncertainty acquired while measuring that variable.

It is possible to interpret the entropy as a measure of uncertainty about an event at frequency f. For spectral entropy, the Fourier transformation method is applied, where it is possible to acquire the power spectral density $p(\omega)$. The power spectral density represents the distribution of power as a function of frequency. Consequently, the normalization of $\mathrm{p}(\omega)$ gives a probability density function. If the definition of Shannon's entropy is applied, it is possible to determine the spectral entropy as:

$$
S P(x)=\sum_{i=f_{1}}^{f_{n}} p\left(x_{i}\right) \cdot \log p\left(x_{i}\right)
$$

where $\left[f_{1}, f_{n}\right]$ is the frequency band. Spectral entropy is usually normalized $\operatorname{SP}(x) / \log N_{f}$ , where $\mathrm{N}_{\mathrm{f}}$ is the number of frequency components in the range $\left[f_{l}, f_{n}\right]$.

The probability law is modelized as the power spectral density of a process, that is:

$$
p(x)=\frac{\sigma^{2}}{\left|1-\sum_{k=1}^{p} a_{k} \cdot e^{-j 2 \pi k x}\right|^{2}}
$$

Spectral entropy, which describes the complexity of a system, is determined in the following way:

1. The spectrum $X\left(\omega_{\mathrm{i}}\right)$ of the signal is calculated.

2. The power spectral density of the signal is calculated in such a way that its amplitude is squared and normalized by the number of bins.

$$
p\left(\omega_{i}\right)=\frac{1}{N}\left|X\left(\omega_{i}\right)\right|^{2}
$$

3. The calculated power spectral density is normalized, so that it is possible to regard it as a probability density function.

$$
p_{i}=\frac{p\left(\omega_{i}\right)^{2}}{\sum_{i} p\left(\omega_{i}\right)}
$$


4. It is now possible to use a standard entropy calculation formula to calculate the spectral entropy.

$$
S P(x)=\sum_{i=1}^{n} p\left(\omega_{i}\right) \cdot \log p\left(\omega_{i}\right)
$$

When boosting the noise signal, the entropy will change if no other processing is carried out. In the beginning, the time range of a measured signal for adaptive spectrogram calculation is very wide. Subsequently this range, however, needs to be reduced, and the degree of reduction depends on the desired results. As the Fourier integral is included in the elementary operations when looking for an appropriate elementary function, the calculation process is very effective. The size of timefrequency interval has the largest impact upon the accuracy of approximation. Narrower intervals ensure better accuracy of representation, but in this case the time of calculation increases. Consequently, it is necessary to find a compromise between the accuracy of approximation and the computation efficiency. It is recommended that the suitable elementary function is selected first and afterwards the parameters needed for spectral entropy are calculated.

\section{Test bench investigations}

The introduced method was applied in the course of measurements on an exemplary multi-plate clutch system for automotive applications. All the measurements were carried out at a test plant at the Institute of Automotive Engineering, Graz University of Technology. A more detailed description of the investigated clutch is available in (Gradwohl, 2017).

The introduced approach of adaptive transform with entropy was used to investigate presence and impact of potential torque oscillations. Due to a restriction of the features of elementary functions, the adaptive spectrogram has been adjusted to a fine adaptive time-frequency resolution, and the time-frequency resolution of the transform is adapted to the specific signal characteristics. It is possible to use Gauss function (impulse) and linear chirp with Gauss window an elementary function. If linear chirps that compose a signal are the result of a linear change in the rotational frequency of a clutch, an adaptive spectrogram can be used in order to determine in which ways a possible frequency modulation is reflected in the time-frequency domain. Adaptive representation is approaching non-linear modulation in the form of a linear combination of functions with linear frequency modulation. Because of this the time for transform calculation is longer, and the quantity of data and the number of cycles required to search for an adequate elementary function are larger.

In the present exemplary investigation, the signal of measured values (torque) had a duration of 2,5 seconds. In an average measurement cycle, 15000 measuring points were sampled. The tested clutch was a dry type with five steel- and four friction plates, leading to eight friction surfaces. 
For comparison purpose, a uniform design of the adaptive spectrograms related to time-frequency transform is defined with a length of the window of 720 points, i.e. $12 \%$ larger than the length of the period of one rotation of the clutch.

The spectrogram can be evaluated on the basis of an average spectrogram that represents an amplitude spectrum of a hybrid adaptive transform of a measured signal, which is based on the observation of individual frequency components. Fig. 2. and 3 show the time-related signal of the torque load of the clutch. Two different measurements have been performed, which consider the influence of new and used friction surfaces. In this way, there is a difference between the clutch that was run in and the clutch that was not run in.

In the Frequency specter in Fig. 4, the frequency components are slightly increased at $160 \mathrm{~Hz}$, but without information about changes in time. Fig. 5 shows the dominant frequency component at $400 \mathrm{~Hz}$. As the amplitude scale is the same in the representation of the two clutch status (one not run in and the other run in), it can be noted that the behaviour of a dry clutch changes considerably after the running-in phase. If the analysis is carried out with the short time frequency transform (Fig. 6 and Fig 7), the difference between spectrograms is very small and the portion of the noise is very large.

It is very interesting to monitor the presence of frequency components, which is typical for frequencies of about $160 \mathrm{~Hz}$ and $400 \mathrm{~Hz}$. This phenomenon is expressed only in the hybrid adaptive spectrogram. In relation to this, spectral entropy of elementary function is calculated and only those elementary functions are selected that have the entropy smaller than previously calculated (Fig. 8).

The new calculated elementary function has entropy that is limited towards the final value (Fig. 9). In such a way, the final selection of the elementary function is obtained more effective (Fig. 10.). Fig. 11 shows the adaptive spectrogram for the first one of the elementary functions for the clutch that was run in. The next new calculated elementary function with smaller entropy is presented in the adaptive spectrogram in Fig. 12. The last and final elementary function which we used for adaptive spectrogram show Fig. 13.

At the same time, it is evident that the collection of energy at individual frequency components in time is fairly present at $160 \mathrm{~Hz}$ in case of the not run in clutch. In Fig. 13 , it is evident in case of the run in clutch that the presence of the frequency component $160 \mathrm{~Hz}$ in time is very small and the dominating frequency component is $400 \mathrm{~Hz}$ with side frequencies, which indicates smoother operation.

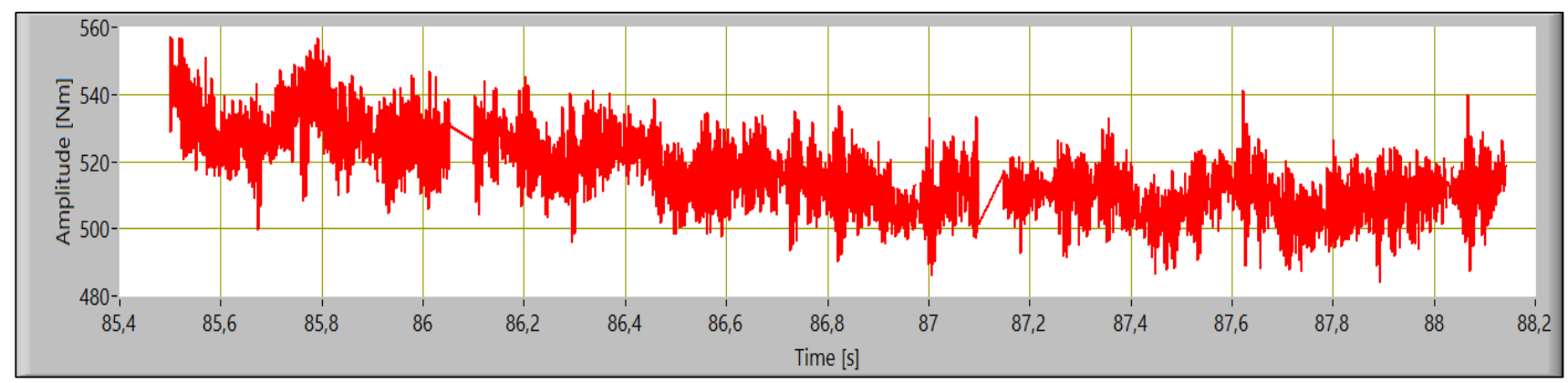

Fig. 2. Time signal of Torque; clutch was not run in 
Belsak, A. \& Hirz, M.: Torsional Vibration of Multi-Plate Clutches Analaysed with...

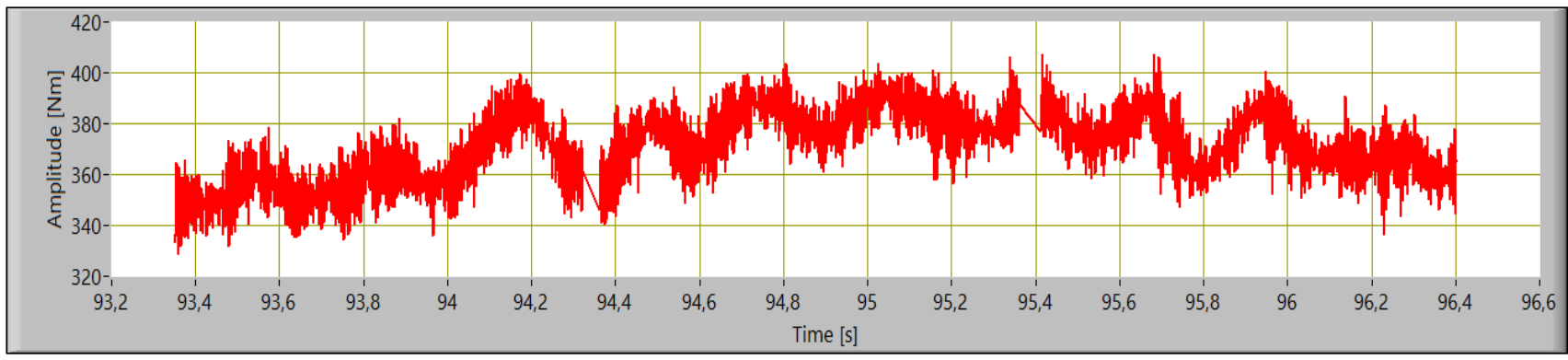

Fig. 3. Time signal of Torque; clutch was run in

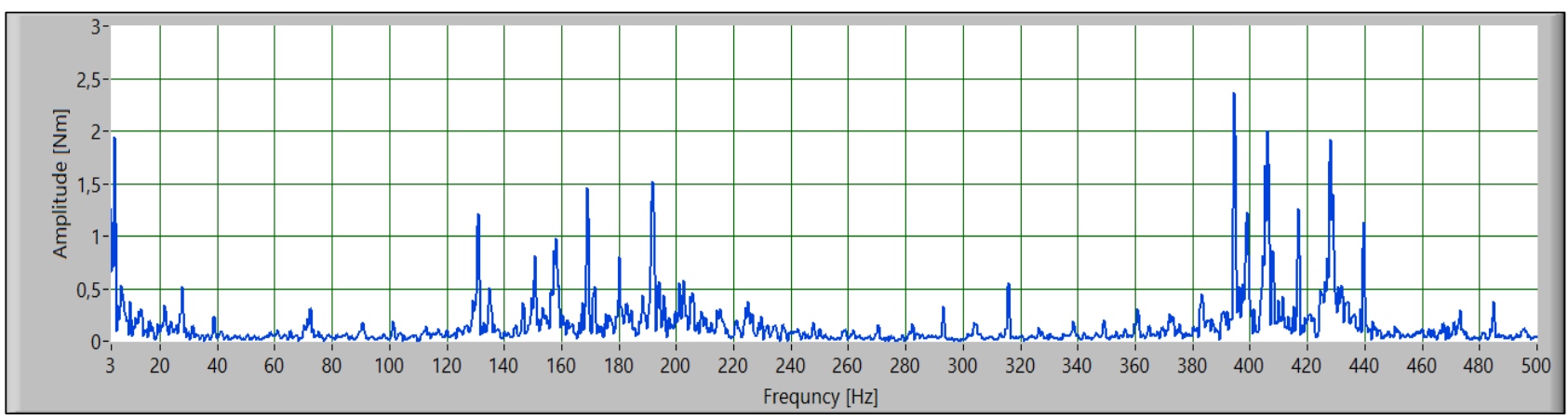

Fig. 4. Frequency specter; clutch was not run in



Fig. 5. Frequency specter; clutch was run in

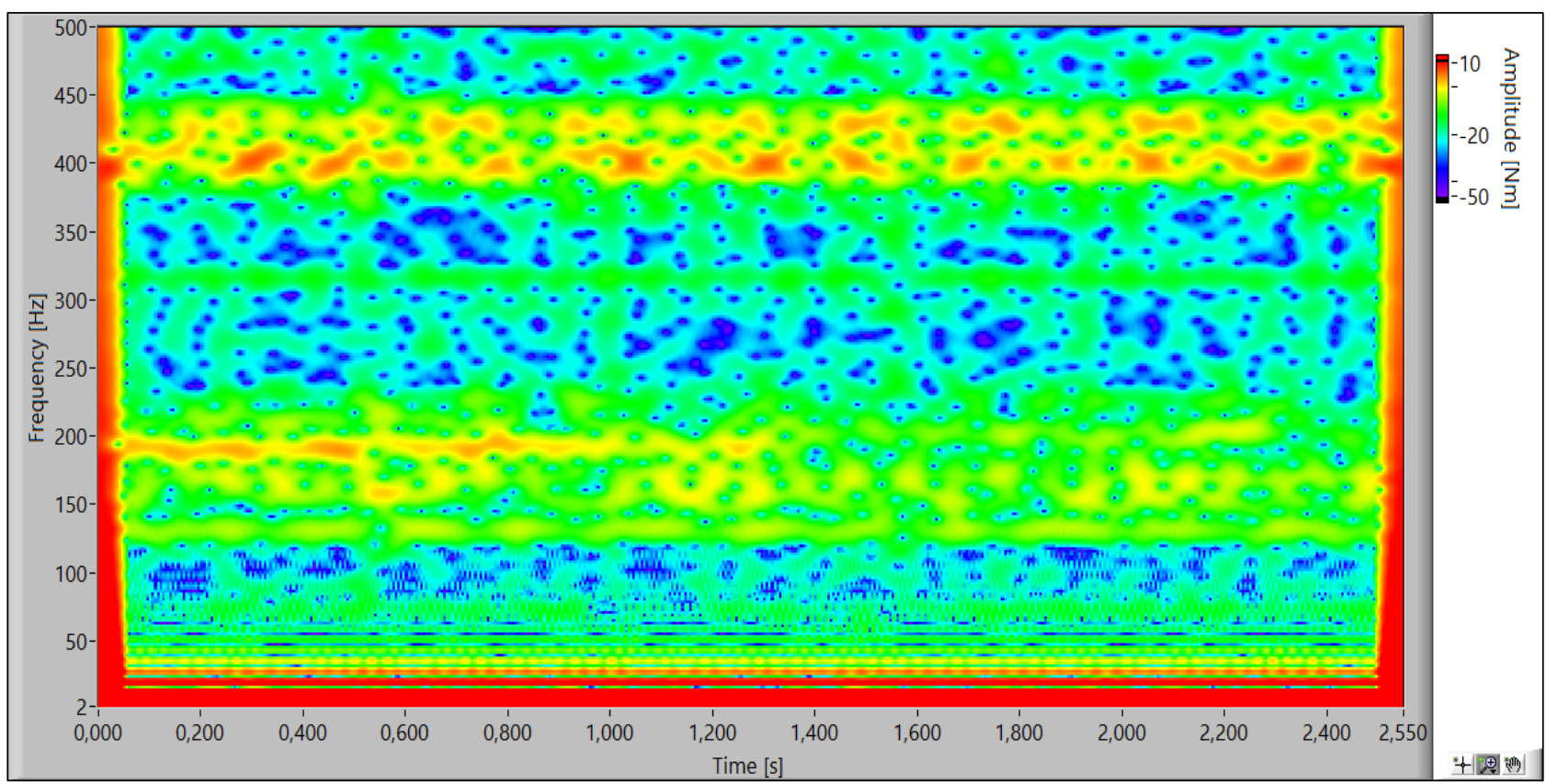

Fig. 6. Short time frequency spectrogram; clutch was not run in 




Fig. 7. Short time frequency spectrogram; clutch was run in

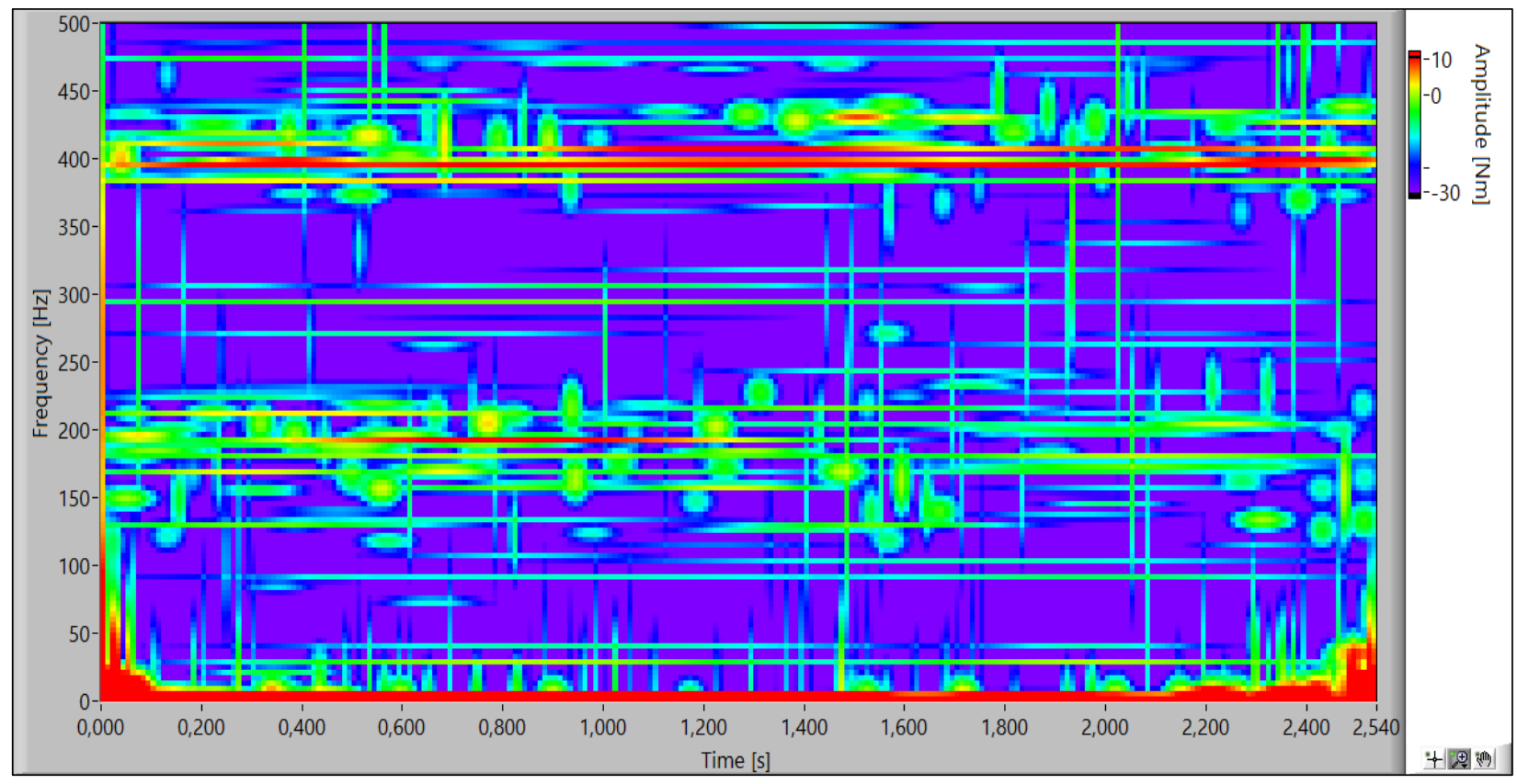

Fig. 8. Time frequency adaptive spectrogram; clutch was not run in and Entropy of elementary functions $h_{p}(t): S P(x)=1,17 \cdot S P(x)$ final 
Belsak, A. \& Hirz, M.: Torsional Vibration of Multi-Plate Clutches Analaysed with...

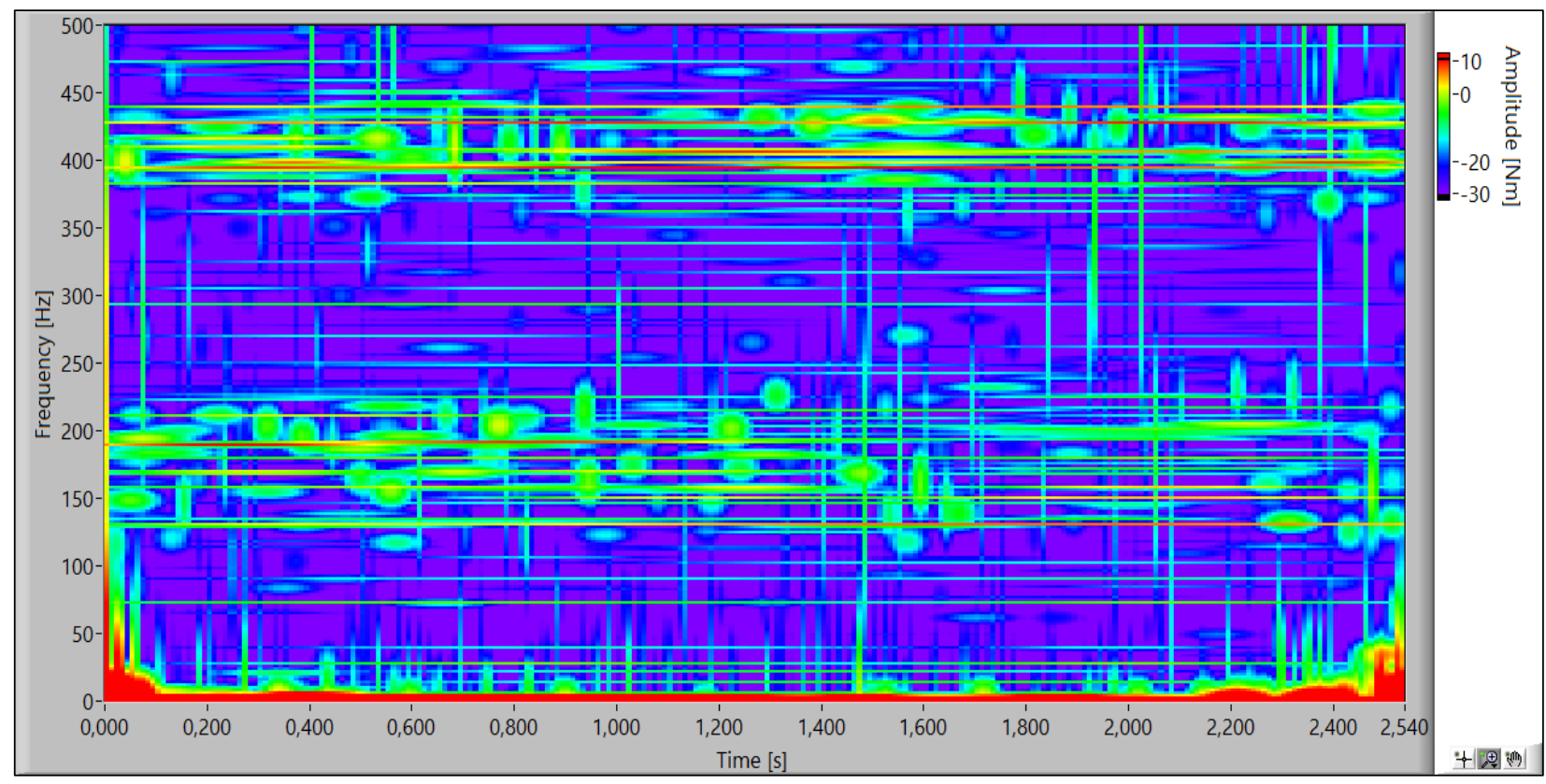

Fig. 9. Time frequency adaptive spectrogram; clutch was not run in and Entropy of elementary functions $h_{p}(t): S P(x)=1,08 \cdot S P(x)$ final

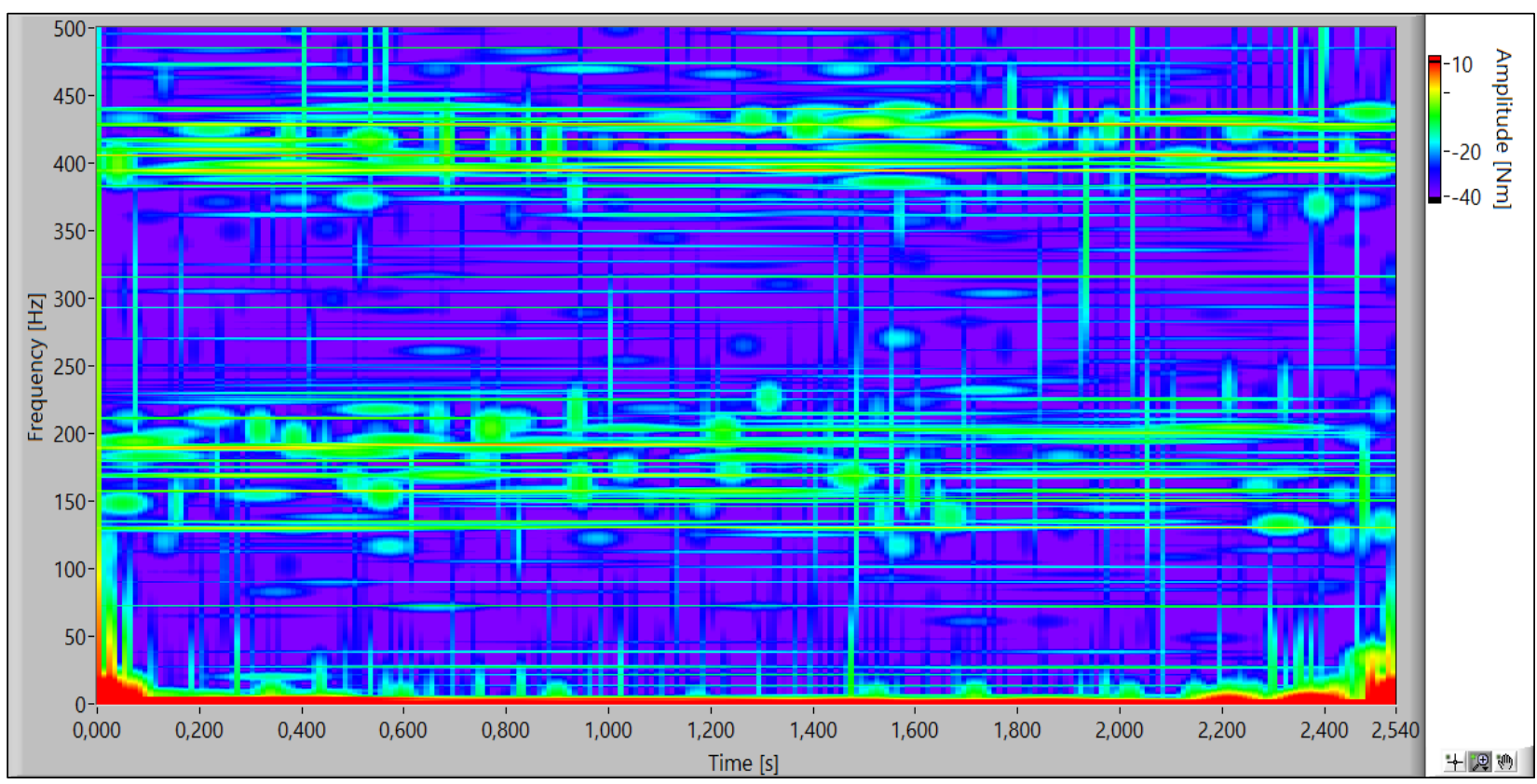

Fig. 10. Time frequency adaptive spectrogram; clutch was not run in and Entropy of elementary functions $h_{p}(t): S P(x)$ final 


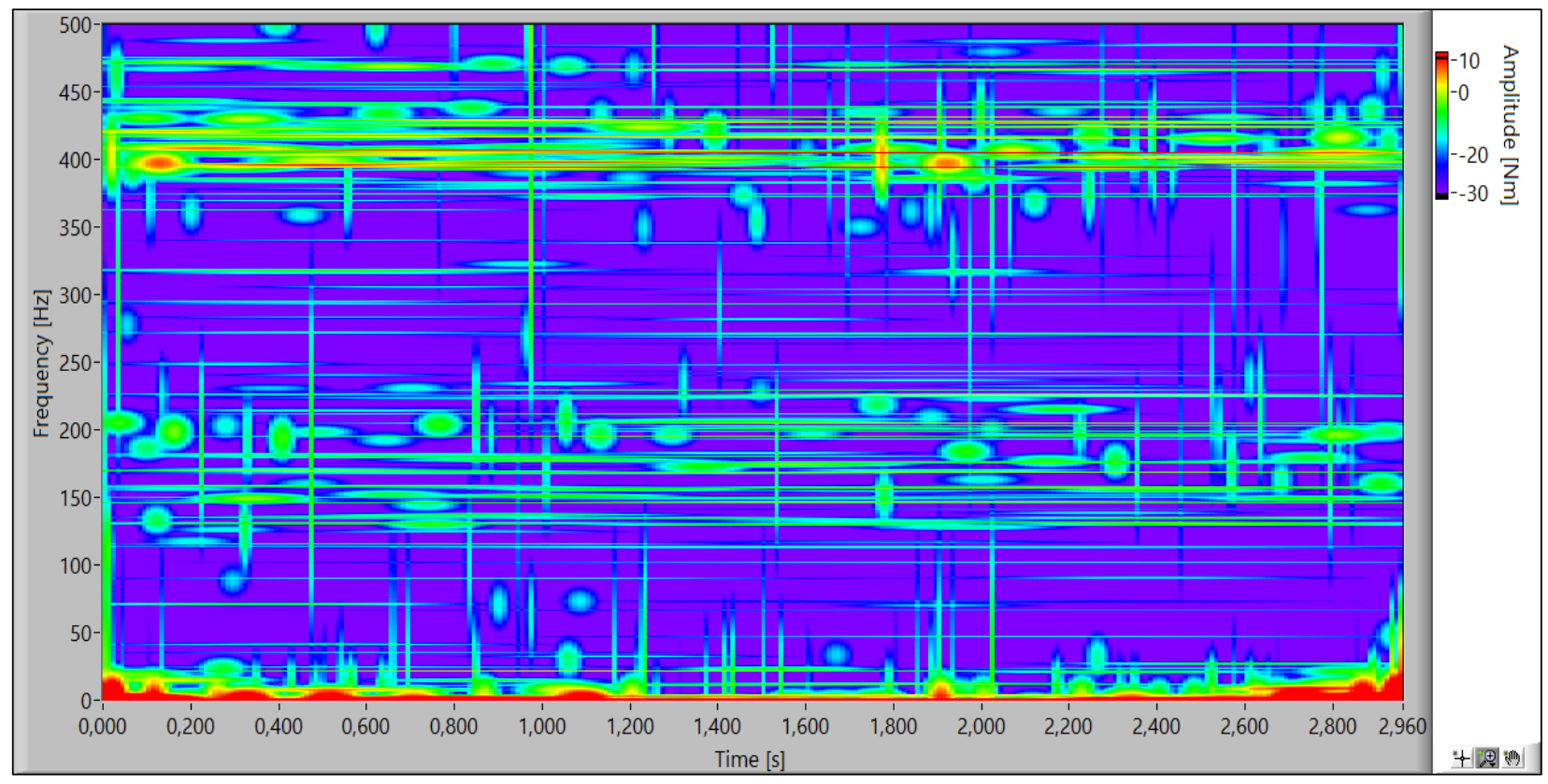

Fig. 11. Time frequency adaptive spectrogram; clutch was run in and Entropy of elementary functions $h_{p}(t): S P(x)=1,11 \cdot S P(x)$ final

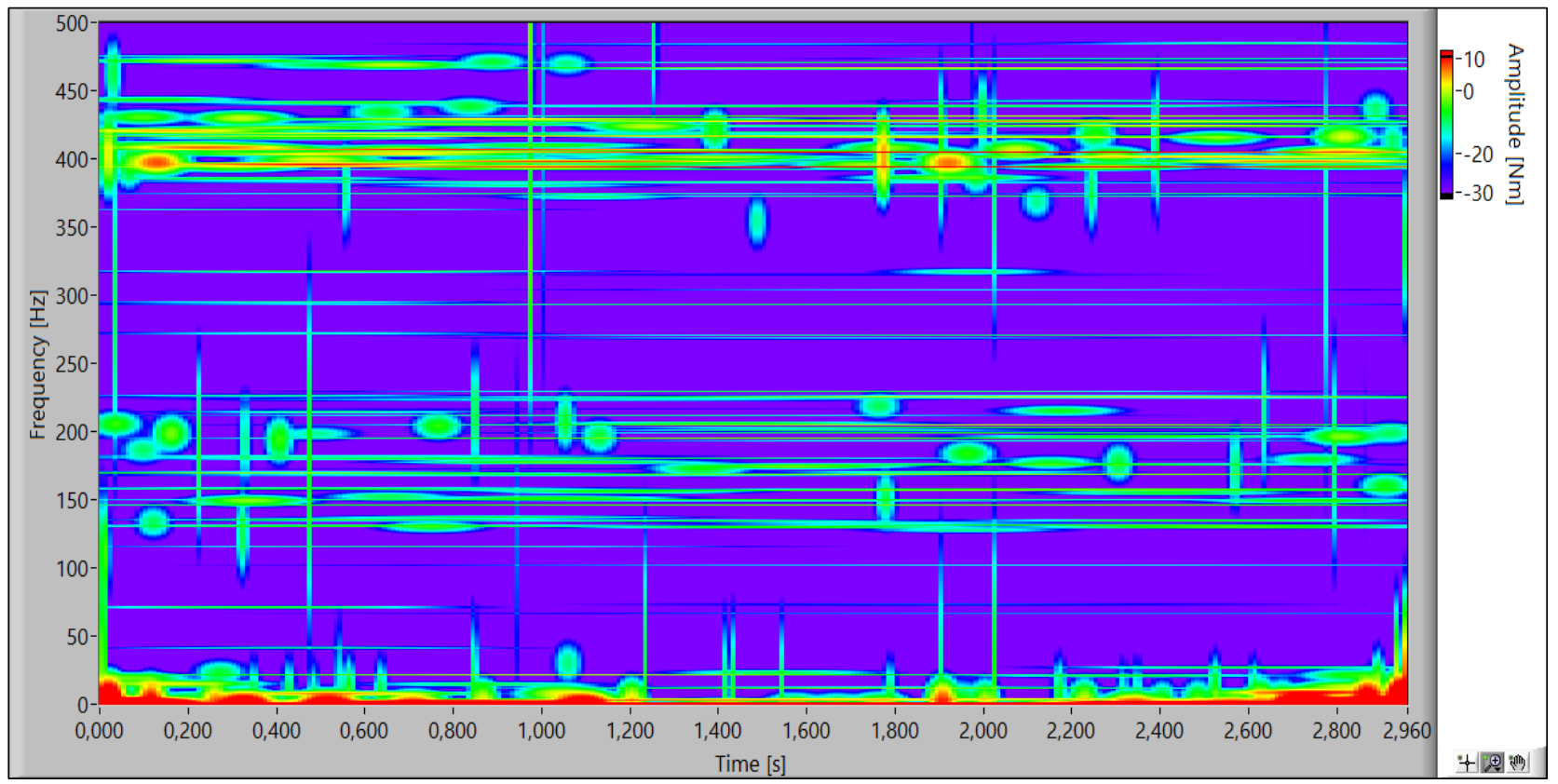

Fig. 12. Time frequency adaptive spectrogram; clutch was run in and Entropy of elementary functions $h_{p}(t): S P(x)=1,06 \cdot S P(x)$ final 
Belsak, A. \& Hirz, M.: Torsional Vibration of Multi-Plate Clutches Analaysed with...

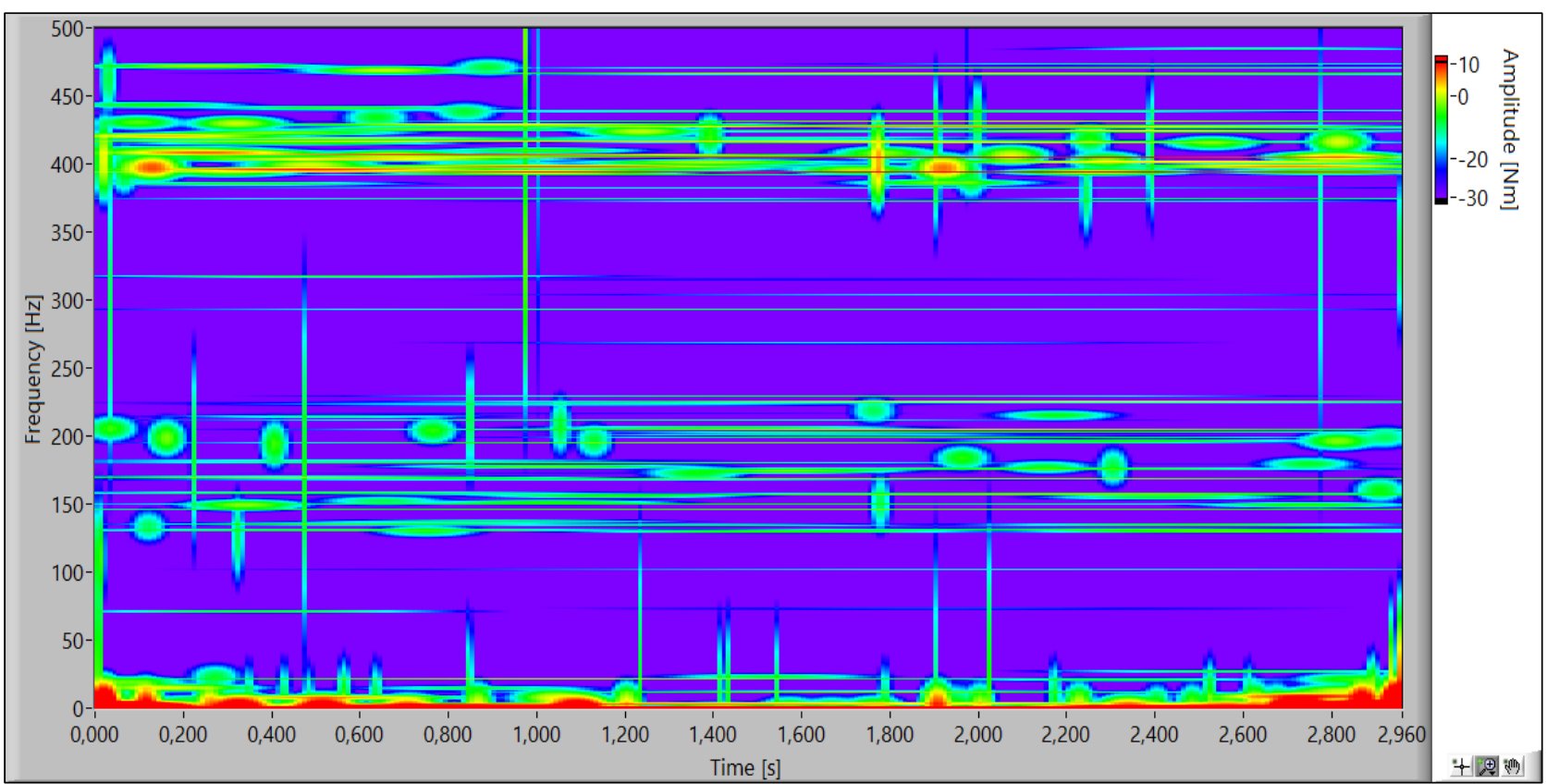

Fig. 13. Time frequency adaptive spectrogram; clutch was run in and Entropy of elementary functions $h_{p}(t)=S P(x)$ final

\section{Conclusion}

The paper presents a new approach of torsional vibration analysis, and applies the method exemplary on an automotive multi-plate dry clutch system. The potential applications of the method are wide spread. Exemplary, it can be used to monitor the life cycle of clutch systems in a more reliable way by using appropriate spectrogram samples and a clear presentation of individual frequency components. In addition to the average spectrum, they represent a criterion that can be used to assess the actual condition of a clutch. By using the hybrid adaptive time-frequency representation, it is possible to predict potential errors and faults effectively, without disseminating a larger quantity of signal energy into the surroundings.

In relation to life cycle design, this represents an adequate method that delivers criteria to monitor the actual condition and vital component parts, which can influence the operational capability in a considerable way. In this way, the introduced method is able to support the enhancement of development processes of clutch systems and contributes to an improvement of functional safety throughout the entire operation phase.

\section{References}

Gradwohl, C.; Belak, A.; Hirz, M. (2017). Dynamic simulation of multi-plate clutches for automotive applications. ASim17-10, pp.1-17

Belsak, A.; Hirz, M. (2018). Torsional vibration analysis of multi-plate clutches for vehicles. V: HORVAT, Marko (ur.), KRHEN, Miljenko (ur.). Conference proceedings, The 8th Congress of the Alps Adria Acoustics Association, 20-21 September 2018, Zagreb, Croatia. Zagreb: Hrvatsko akustičko društvo (HAD) / Acoustical Society of Croatia (ASC)., pp. 59-63 
Ompusunggu, P.; Sas P.; Van Brussel H. (2013). Modeling and simulation of the engagement dynamics of a wet friction clutch system subjected to degradation: An application to condition monitoring and prognostics, Mechatronics, 23, pp. 700-712 Dolcini, P. J.; Canudas, C.; Béchart, B. H. (2010). Dry Clutch Control for Automotive Applications, Springer-Verlag London

Orthwein, W. C. (2004). Clutches and Brakes, Marcel Dekker

Butlin, T.; Woodhouse, J. (2009) Sensitivity studies of friction-induced vibration, Int. J. Vehicle Design, Vol. 51, Nos. 1/2, pp. 111-129

Popov, V. L. (2010). Contact Mechanics and Friction, Springer-Verlag Berlin Heidelberg

Gang, C. (2014). Handbook of friction-vibrations interactions, Woodhead Publishing Sergienko V. P.; Bukharov, S. N. (2015). Noise and Vibration in Friction Systems, Springer International Publishing Switzerland

Ouyang, H.; Motterhead, J. E. (2001). Unstable travelling waves in the friction-induced vibrations of disc, Journal of Sound and Vibration 248(4), pp. 768-779

Qiao, S. L.; Ibrahm, R. A. (1999). Stohastic dynamic of system with friction-induced vibration, Journal of Sound and Vibration 223(1), pp. 115-140

Centea, D.; Rahnejat, H.; Menday, M.T. (2001). Non-linear multi-body dynamic analysis for the study of clutch torsional vibrations (judder), Applied Mathematical Modelling 25, pp. 177-192

Chengwu, D., Rajendra, S. (2009). Forced vibrations of a torsional oscillator with Coulomb friction under aperiodically varying normal load, Journal of Sound and Vibration, 325, pp. 499-506

He, L.; Li, L.; Yu, L. Y.; Song, J. (2011). Multi-state control strategy of starting for a wet friction clutch via a fuzzy logic algorithm; International Journal of Automotive Technology, Vol. 12, No. 4, pp. 537-544

Lingestena, N.; Marklunda P.; Höglunda, E.; Lunda, M.; Lundinb, J.; Mäkib, R. (2012). Apparatus for continuous wear measurements during wet clutch durability tests, Wear 288, pp. 54-61

Pinte, G.; Depraetere, B.; Symens, W.; Swevers, J.; Sas, P. (2010). Iterative learning control for the filling of wet clutches, Mechanical Systems and Signal Processing 24, pp. 1924-1937

Meng, F.; Chen, H.; Zhang, T.; Zhu, X. (2015). Clutch fill control of an automatic transmission for heavy-duty vehicle applications, Mechanical Systems and Signal Processing 64-65, pp. 16-28

Butlin, T.; Woodhouse, J. (2009). Sensitivity studies of friction-induced vibration, Int. J. Vehicle Design, Vol. 51, Nos. 1/2

Ibrahim, R.A.; Madhavan, S.; Qiao, S.L.; Chang, W.K. (2000). Experimental investigation of friction-induced noise in disc brake systems, International Journal of Vehicle Design, Vol.23 No.3/4, pp. 218-240

Zamora, P.; Lagunas, M. A. (1995). New approaches in nonlinear signal processing: Estimation of the PDF function by spectral estimation methods, Proc. IEEE-Athos Workshop Higher-Order Stat., pp. 204-208.

Zamora P.; Lagunas, M. A. (1999). Fourier models for nonlinear signal processing, Signal Process., vol. 76, no. 1, pp. 1-16 
Belsak, A. \& Hirz, M.: Torsional Vibration of Multi-Plate Clutches Analaysed with...

Kay, S. (1998). Model-based probability density function estimation, IEEE Signal Processing Lett., vol. 5, pp. 318-320

Bercher, J. F.; Vignat, C. (1999). Estimating the entropy of a signal with applications, Proc. ICASSP, Phoenix, AZ

Qian, S.; Chen, D. (1996). Joint Time-Frequency Analysis, Prentice Hall

Mertins, M. (1999). Signal Analysis, John Wiley \& Sons Inc., New York

Bercher, J. F.; Vignat C. (2000). Estimating the Entropy of a Signal with Applications, IEEE Transaction on signal processing, Vol. 48, No. 6

Omelchak, A.; Fecak, S. \& Idrisova, U. (2016). Dynamic Processes in a Machine-Tool at High-Speed Machining, DAAAM International Scientific Book 2016, Vol. 15, ISSN 1726-9687, ISBN: 978-3-902734-09-9, Editor: B. Katalinic, DAAAM International Vienna, Vienna

Dokoupil, J. \& Pivonka, P. (2011). Adaptive Nonlinear Model Predictive Control Based on Wiener Model, DAAAM International Scientific Book 2011, Vol. 10, ISSN 1726-9687, ISBN: 978-3-902734-84-1, Editor: B. Katalinic, DAAAM International Vienna, Vienna

Zeng, X. T.; Meng, G. Y. \& Zhou, J. H. (2018) Analysis on the Pose and Dynamic Response of Hydraulic Support under Dual Impact Loads, International Journal of Simulation Modelling, Vol. 17, No. 1, pp. 69-80, ISSN 1726-4529

Djemana, M. \& Hrairi, M. (2016) Modelling and Simulation of Impedance-Based Damage Monitoring of Structures International Journal of Simulation Modelling, Vol. 15, No. 3, pp. 395-408, ISSN 1726-4529 